\title{
PLANIFICACIÓN DE UNA UNIDAD DIDÁCTICA: EL ESTUDIO DEL MOVIMIENTO
}

\author{
GARCía ARQUeS, J.J., PRO BUENO, A. y SAURA LlAMAS, 0 . \\ Departamento de Didáctica de Ciencias Experimentales. Facultad de Educación. \\ Universidad de Murcia.
}

\section{SUMMARY}

In this study we attempt to apply an outline of Teaching Units for the study of Cinematics at Secondary Level. Having analysed the teaching problems indicated by teachers, the scientific contents involved and the methodological problems, a sequence of learning contents is established from a creative construction perspective.

\section{INTRODUCCIÓN}

Los fundamentos para la acción didáctica de los profesores en el aula fueron descritos en uno de nuestros anteriores trabajos (Valcárcel et al. 1990). Entonces señalábamos que, en el proceso de planificación đe cualquier intervención docente, el profesorado -individual o colectivamente- pone en juego sus conocimientos científicos y didácticos y, sobre todo, su práctica educativa (Fig. 1). Desde esta perspectiva consideramos fundamental incidir en todos los elementos que pueden aportar ideas a los profesores de cara a las decisiones que toma, explícita o implícitamente, en un momento tan importante de su labor diaria.

Además, esta importancia se ve «reforzada» con la puesta en práctica de un nuevo currículo oficial, en el que se señala un marco de referencia, pero se deja a los equipos docentes de los centros la distribución y secuenciación de los contenidos y al profesor la adaptación de los mismos a las singularidades de sus alumnos (MEC, 1989). Esto, por lo menos teóricamente, debe incidir en Ia necesidad de romper con la inercia de la homogeneidad de temarios, programas, métodos... con alumnos y contextos diferentes. En cualquier caso, da una mayor responsabilidad al profesorado y lógicamente sería deseable que se les facilitaran instrumentos para hacer frente a esta competencia.
En este contexto podríamos enmarcar nuestro trabajo: analizar la problemática que tiene la enseñanza y el aprendizaje (E/A) de un tema determinado, extraer consecuencias didácticas para su trabajo en el aula y plantear una propuesta điđáctica concreta -aunque no sea la única posible-que se fundamente en todo lo anterior. AI respecto habria que decir que creemos que hay aportaciones suficientemente importantes en la investigación e innovación educativas de la didáctica de las ciencias para ir dando respuestas a los interrogantes que todos nos hacemos antes, durante y después de entrar en el aula.

Se han diseñado, ensayado y evaluado algunas tunidades didácticas tratando de responder a estas necesidades (Hierrezuelo et al. 1991, Seminario Física y Química 1990, Valcárcel et al. 1990). Sin embargo, en este artículo quisiéramos incidir to sólo en una propuesta específica sino sobre el proceso seguido para su fundamentación, ya que pensamos que es extrapolable a otras situaciones.

En efecto, los fundamentos que usamos en nuestras propuestas son:

a) perspectiva del profesorado en ejercicio; 
b) análisis científico (conceptual y proceđimental) de contenidos;

c) estudio de la problemática del aprendizaje de dichos contenidos;

d) selección de actividades y estrategias didácticas.

\section{EL ESTUDIO DE LA CINEMÁTICA DESDE LA PERSPECTIVA DEL PROFESORADO EN EJERCICIO}

Uno de los elementos que puede servir de referencia en temas que habitualmente hayan sido impartidos en nuestras aulas es, sin duda, la reflexión sobre la propia práctica diaria. Pero quedaría incompleta si no la contrastamos con la de otros profesores que también se plantean diariamente los mismos retos que nosotros. Pensamos que ésta puede ser una asignatura pendiente en estos momentos de la Reforma: el intercambio de experiencias, problemas y necesidades reales entre los profesionales en ejercicio.

Para ver la perspectiva de otros compañeros respecto a Ia problemática que presenta el estudio de la cinemática, se diseñó un instrumento de recogida de información taI como aparece en el anexo; posteriormente se completó con una entrevista que, en muchos casos, resultó muy clarificadora de cara a la interpretación de Ios resultados. Fue pasado a 15 profesores de estos niveles educativos ( 7 de EGB y 8 de EEMM) que, si bien no se pueden considerar representativos estadísticamente de la población de origen, sí presentaban un perfil profesional y una actitud de colaboración muy apropiadas para las intenciones de nuestro trabajo. Sólo exponemos una síntesis de los resultados obtenidos.

\section{Respecto al nivel donde deben ser estudiados los contenidos}

- Se advierte una tendencia general a abordar en los primeros niveles las nociones de reposo y movimiento, sistema de referencia, trayectoria y desplazamiento, de forma más intuitiva que sistematizada. El único concepto que aparece en el primer ciclo es el de velocidad media, lo cual parece coherente tanto desde una perspectiva científica como didáctica.

- Son mayoría los que incluyen las representaciones gráficas $\circ$ el uso de las ecuaciones representativas del movimiento uniforme en el primer ciclo (suele ser habitual hoy que en el ciclo superior de EGB se impartan estos contenidos).

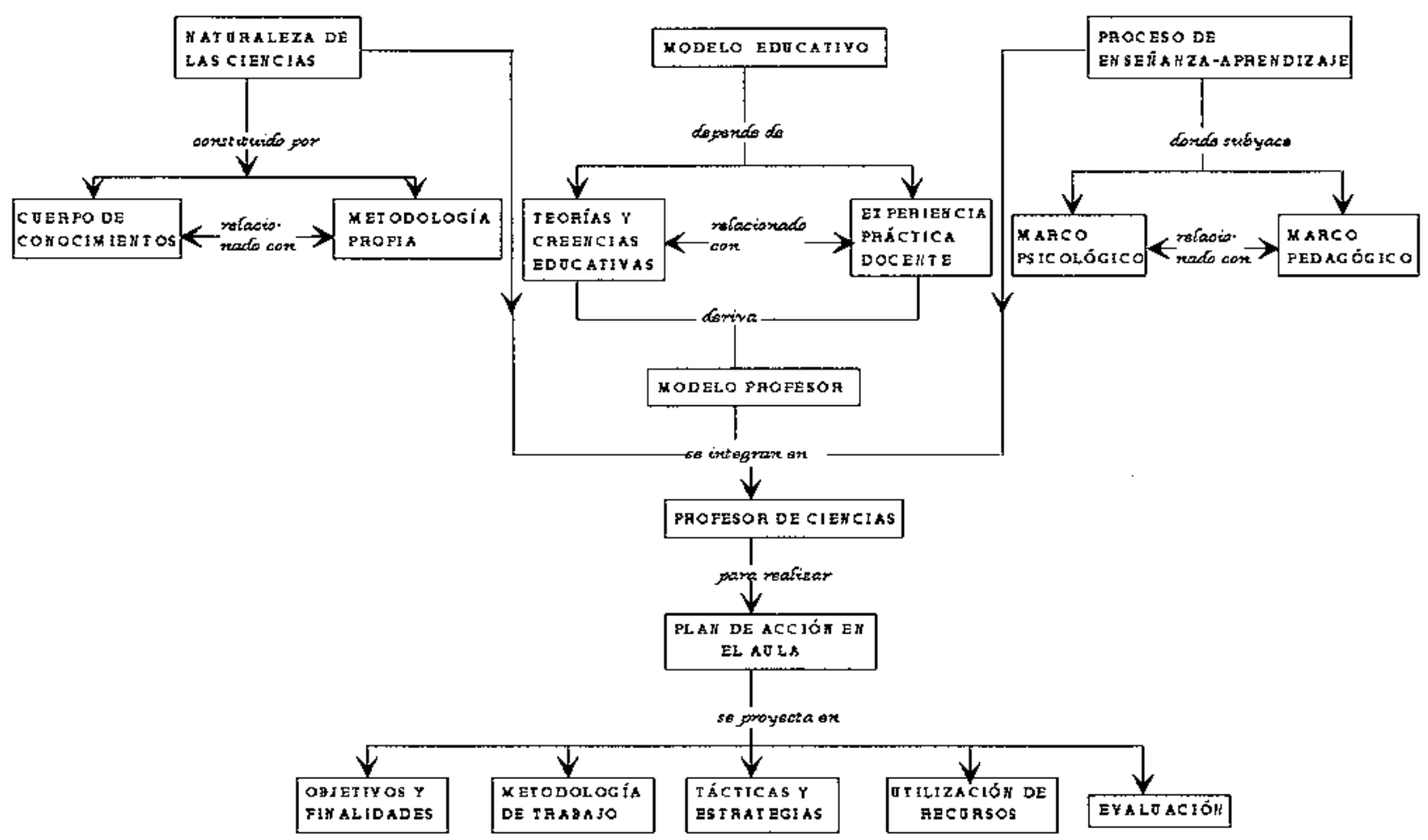


- Un número importante de profesores secuencia antes el estudio de la aceleración media que el de la velocidad instantánea $(5 / 15)$. En algunos casos $(2 / 15)$, se habla antes de las ecuaciones representativas del movimiento uniformemente acelerado que de la velocidad o aceleración instantánea.

- Hay una cierta unanimidad a la hora de considerar el estudio del movimiento circular y ondulatorio inadecuados para la educación secundaria obligatoria (ESO); una percepción similar se tiene respecto a la composición de movimientos.

Las justificaciones o criterios aludidos por los profesores en sus decisiones son variados (el esquema conceptual del alumno, su capacidad de razonamiento y sus conocimientos matemáticos, mi propia experiencia, el grado de dificultad, la motivación que produce y los intereses de los alumnos...). Sin embargo, cuando indagamos en sus respuestas durante la entrevista posterior, parece debido más a una inercia docente que a otra causa.

\section{Respecto a la importancia de los contenidos en estos niveles}

- Existen contradicciones entre algunas respuestas dadas en el ítem anterior y algunas afirmaciones de los profesores entrevistados; incluso, hay quienes señalan que algunos contenidos son muy importantes en estos niveles y previamente consideraban que no debieran ser estudiados (4/15).

- Se «sobrevalora» la importancia de las ecuaciones representativas de los movimientos, por delante algunas veces de conceptos más «físicos» (velocidad instantánea o media, por ejemplo), lo que, junto a las características del módulo de cinemática, puede llevar a incidir «excesivamente» en los formulismos y en la resolución de ejercicios.

- Se aprecia una heterogeneidad importante en las elecciones de los conceptos más y menos relevantes; esta circunstancia pueđe resultar desorientadora para el alumno si no se unifican los criterios entre los profesores, sobre todo si pertenecen al mismo centro.

\section{Respecto a las difícultades didácticas de los contenidos}

Hay un conjunto de dificultades didácticas según los profesores que suelen ser habituales en el desarrollo de este módulo:

- confusión entre dirección y sentido en las magnitudes vectoriales $(5 / 15)$;

-problemas con la utilización de los sistemas de referencia (5/15);

- confusión entre velocidad media e instantánea (igual con la aceleración) (6/15);
- dificultades para conceptualizar la aceleración (4/15); sobre todo, sus componentes intrínsecas (3/15);

- identificación de conceptos con fórmulas (la velocidad es $v=e / t)(6 / 15)$;

- falta de hábito en el uso de gráficas (5/15).

En líneas generales se puede decir que existe un salto importante desde el estudio del movimiento rectilíneo y uniforme al movimiento uniformemente acelerado (aparición del concepto de aceleración). Un segundo momento, más complejo aún, se produce al estudiar movimientos no rectilíneos (utilización del carácter vectorial de forma sistematizada).

\section{Respecto a las propuestas metodológicas utilizadas}

De forma genérica se dice en el cuestionario que los recursos más utilizados son las explicaciones del profesor y la realización de problemas; también se indica el uso de experiencias de laboratorio para abordar determinadas unidades conceptuales. En la entrevista posterior se puso de manifiesto la escasa telación entre el desarro110 «teórico» y "práctico» (a pesar de la idealización que se hace del trabajo de laboratorio) y la confusión entre ejercicios numéricos y la resolución de problemas. La secuencia más usual era: teoría - ejercicios - prácticas.

En cuanto a las propuestas concretas se presentaron algunos materiales ya editados. Algunos hablaban de estrategias de descubrimiento guiado en las hojas de trabajo de laboratorio e, incluso, uno aludía a un programa-guía. Casi todos decían que usaban con cierta asiduidad técnicas de trabajo en grupos. Cuando ampliamos la información nos dio la impresión de que se había contestado más lo deseado que lo que habitualmente hacían; de hecho se insistió mucho en las «clásicas dificultades» de la puesta en práctica: número de alumnos, escasez de material, locales inapropiados, falta de tiempo para la preparación de las sesiones, etc.

Hay que destacar, en cualquier caso, que los resultados corresponden a una muestra de profesores que podríamos caracterizar por su preocupación profesional, por una cierta formación adquirida en cursos, jornadas, seminarios... y, sobre todo, por sus años de experiencia. Sería necesario conocer mejor, aunque no sea el objetivo de este trabajo, cómo trasfieren sus concepciones aI aula (qué modelo utilizan para el aprendizaje de los alumnos, qué contenidos procedimentales y actitudinales consideran y cómo se secuencian, si el trabajo en grupos está orientado a la suma de opiniones o a la explicitación y al contraste de ideas entre los alumnos...).

Desde un punto de vista científico, habría que resaltar que algunas de las experiencias planteadas tienen una difícultad importante en la medición si se utilizan cronómetros «normales», ya sea de los propios alumnos (digitales) como de los que se suministran con los equipos didácticos de los centros de estos niveles educativos. También parece complicado el estudio del movimiento 
uniforme en una superficie horizontal, sobre todo si hay que controlar varios montajes a la vez para conseguir una aproximación que no induzca a errores a los alumnos. Por último, se observa la indistinta utilización de conceptos como velocidad instantánea y velocidad media en el texto, en las representaciones gráficas, etc. en sus guiones de laboratorio.

\section{ANÁLISIS CIENTÍfICO DE LOS CONTE- NIDOS}

Otro de los fundamentos de cualquier propuesta đidáctica en ciencias deriva de los propios conocimientos científicos implicados y de la percepción que tengamos de la naturaleza de dicho conocimiento. Creemos que la profundización en los contenidos de enseñanza no sólo es una exigencia obvia del profesorado (no se enseña to que no se sabe) sino que, además, da soluciones didácticas a muchos de los problemas planteados en la delimitación, selección y secuenciación de los contenidos objeto de enseñanza. Dada nuestra percepción de los mismos, tendremos que realizar el análisis conceptual y procedimental consiguientes.

Figura 2

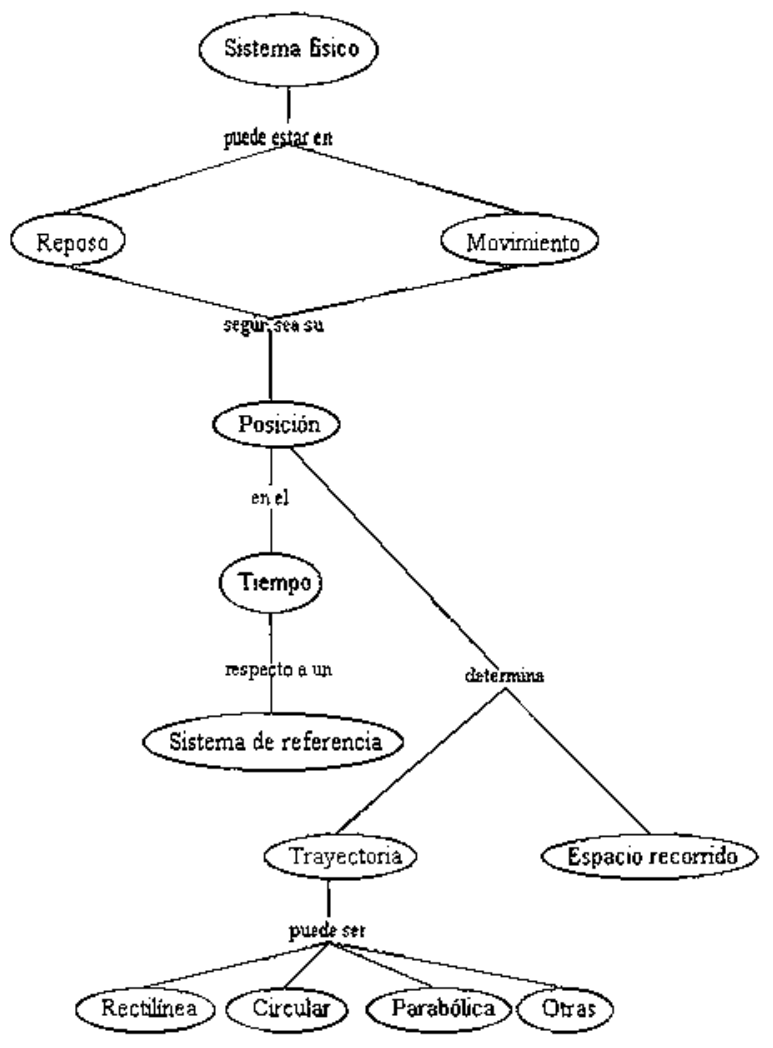

\section{Análisis del marco conceptual}

Para realizar el análisis de los contenidos científicos, hemos distribuido casi todas las unidades mencionadas en el anexo en tres esquemas conceptuales (Novak y Gowin 1988). El primero (Fig. 2) enmarca la intencionalidad del estudio de la cínemática: el reposo y el movimiento de las partículas y de los sistemas físicos.

Sin duda, los conceptos de reposo y de movimiento son los más relevantes de este módulo aunque ambos se encuentran mediatizados por el de sistema de referencia. $\mathrm{Al}$ respecto habría que indicar que el tema del movimiento relativo puede resultar complejo didácticamente como veremos más adelante.

En la figura 3 aparece el marco conceptual que sustenta el estudio de la cinemática. Se puede apreciar cómo los conceptos de velocidad y aceleración constituyen el armazón fundamental en esta parte de la física. El hecho de que algunos de ellos aparezcan en un mismo nivel jerárquico (velocidad - aceleración, velocidad media velocidad instantánea...) no presupone ninguna secuencia organizativa, como ya veremos.

En efecto, existen unos condicionamientos de tipo didáctico-no científico- que impiden conceptualizar completamente, por ejemplo, la velocidad instantánea hasta que el alumno no sea capaz de utilizar algunos operadores matemáticos. Todo ello se hace más evidente si pretendemos usar estrategias metodológicas de tipo experimental donde las extrapolaciones de datos o las inferencias de resultados están sustentadas en valores inexactos, construcciones gráficas aproximadas... No obstante, es conveniente introducir el término aunque sea utilizando los conocimientos próximos del alumno.

En cualquier caso parece necesario comenzar con el concepto de velocidad media, insistiendo en el «apellido» para evitar errores bastante generalizados (la velocidad es siempre espacio partido por tiempo). Análogamente habrá que empezar por trayectorias que no precisen de la utilización đel carácter vectorial para evitar dificultades añadidas al proceso de conceptualización.

Un razonamiento similar al empleado en la velocidad se podría realizar con la aceleración, con el agravante de su dependencia respecto a una magnitud medida indirectamente de manera que las argumentaciones anteriores se duplican. El estudio de las componentes intrínsecas requiere de un tratamiento vectorial con todo lo que implica de cara a una mayor complejidad cognitiva y, por ello, no lo tocamos.

En líneas generales, pensamos que no deberían obviarse, sobre todo en los primeros niveles, los aspectos intuitivos y cualitativos frente a un excesivo operacionalismo matemático. Por otro lado, creemos que es importante que el alumno sea capaz de explicar la coherencia de sus resultados numéricos, de interpretar signos y convencionalismos cuantitativos, de ser crítico con sus hallazgos, sean experimentales o no...; y la cinemática permite abordar toda esta problemática, indudablemente formativa. 
Figura 3

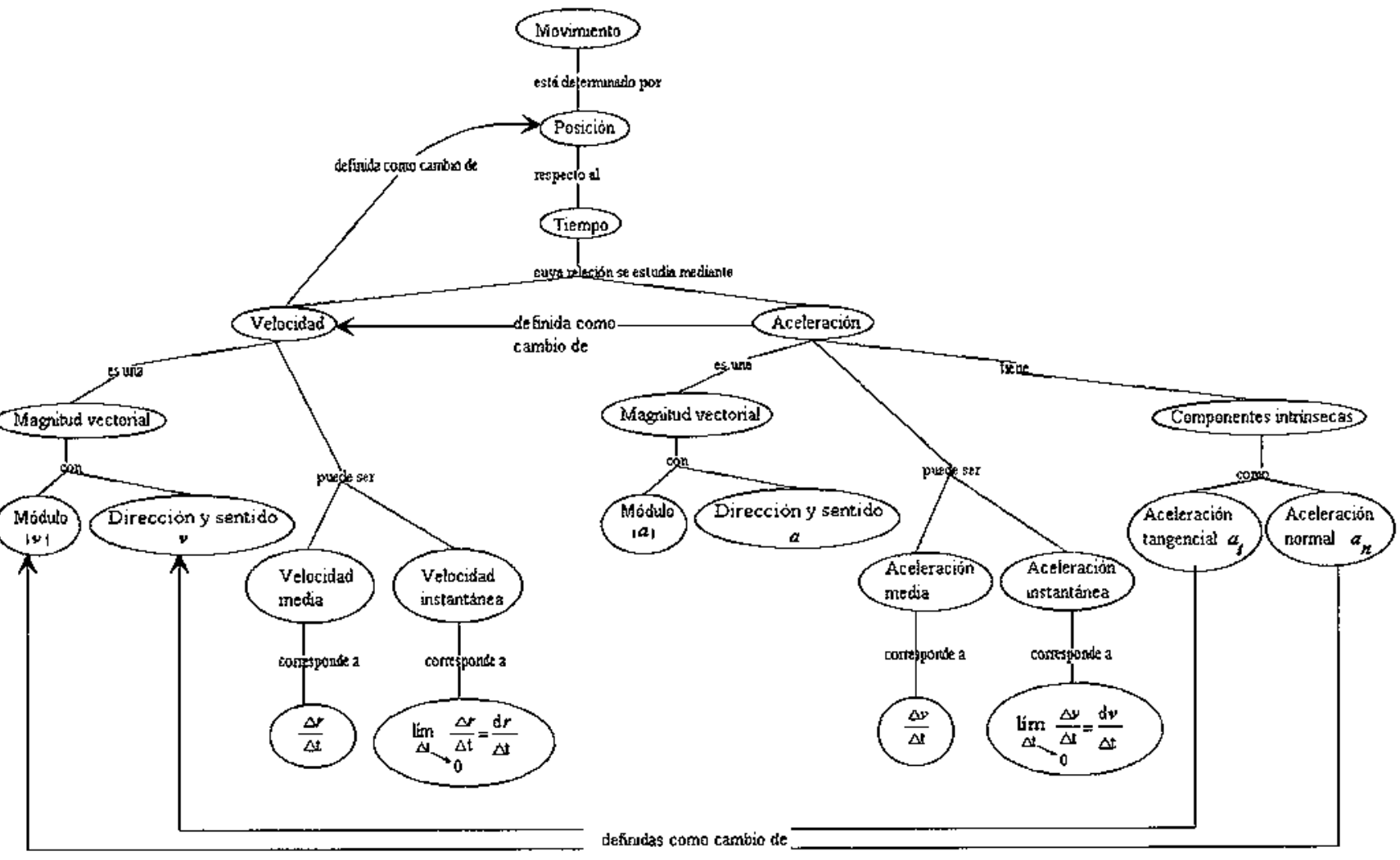

Figura 4

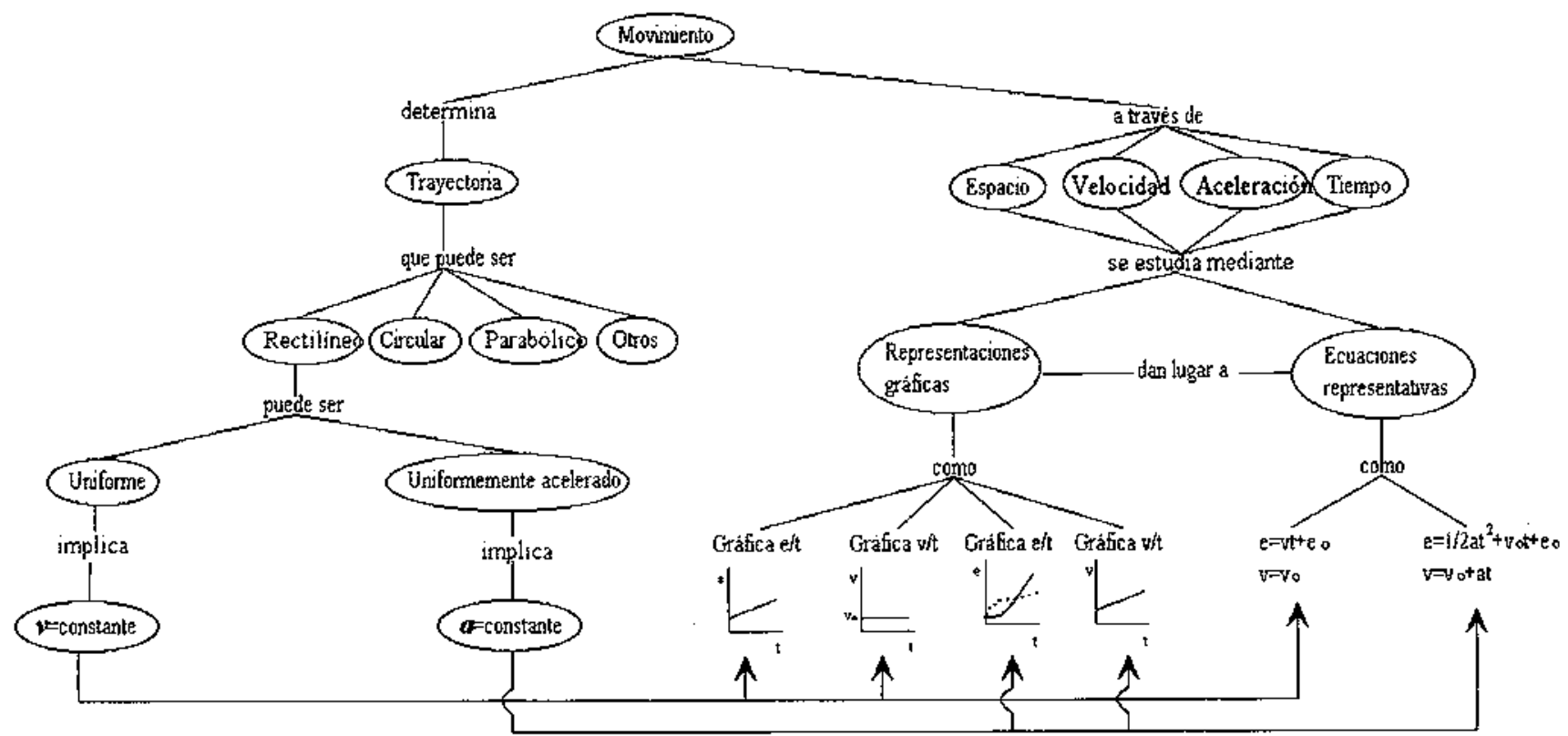


El tercer esquema (Fig. 4) podría considerarse como un complemento de los anteriores. Hemos querido resaltar que en el estudio del movimiento de las partículas o de Ios sistemas hay dos instrumentos matemáticos que se ponen al servicio del mismo: Ias representaciones gráfi cas y las ecuaciones representativas.

Quizás, muchos profesores insistan tanto en estos contenidos que trasmiten una percepción equivocada de esta parte de la física. No obstante, no es un problema científico de los contenidos del módulo sino un plantea* miento didáctico poco coherente con lo que se pretende.

\section{Análisis de los contenidos procedimentales}

En la línea del trabajo de Sánchez y Valcárcel (1993) para realizar un análisis de los contenidos procedimentales creemos interesante profundizar en las afirmaciones de conocimiento implícitas en nuestros mapas anteriores. Las más relevantes serían:

- Un sistema físico está en reposo o en movimiento según su posición en el tiempo respecto a un sistema de referencia.

- La velocidad es una magnitud que representa cl cambio de la posición de una partícula o un sistema físico en el tiempo, respecto a un sistema de referencia.

- La aceleración como cambio del vector velocidad en el tiempo.

- El movimiento rectilíneo y uniforme como aquél que tiene una velocidad constante en módulo, dirección y sentido.

- El movimiento rectilíneo y uniformemente acelerado como aquél que tiene una aceleración constante en módulo dirección y sentido (aceleración tangencial constante y aceleración normal igual a cero).

Usando la técnica de la V de Gowin (Novak y Gowin 1988) para relacionar el marco procedimental con el conceptual obtuvimos las representaciones correspondientes. En las figuras 5 y 6 aparecen sólo dos de ellas para no alargar excesivamente la exposición de nuestro trabajo. Del análisis completo se pueden extraer las siguientes conclusiones:

- Esta unidad didáctica permite poner en juego un gran conjunto de procedimientos como contenidos de enseñanza (no sólo como estrategias o recursos del profesor): descripción de observaciones, realización de montajes, predicción de resultados, realización de medidas y valoración de errores, representación e interpretación đe gráficas, valoración de resultados matemáticos, establecimiento de conclusiones, etc.

- El marco teórico, aunque sea común, no debe obviarse en el desarrollo de los contenidos procedimentales.

- Se debe y se puede aprovecbar la proximidad que tienen algunos conceptos del lenguaje cotidiano (como la velocidad y la aceleración) para, a partir de ello, lograr la formalización académica de los mismos.

- El tema se presta a seguir reiteradamente la secuencia «observación - recogida de datos - análisis de los mismos - ley experimental», cuestión que no debe generalizarse a otras unidades de las ciencias.

- Resulta extremadamente útil el trabajo en grupos y la elaboración de informes, dada la supeditación de las conclusiones a la recogida de datos.

Es muy importante, desde nuestra perspectiva, que el alumno perciba «un todo» en el aprendizaje de estos contenidos (conceptuales y procedimentales) y que el profesorado sea consciente de que igual que los primeros precisan de un tiempo para ser aprendidos, los segundos también precisan de una dedicación temporal, aun a costa de «impartir menos materia».

\section{PROBLEMÁTICADIDÁCTICA DEL MÓDULO DE CINEMATICA}

No sería coherente con nuestra concepción del proceso de E/A de las ciencias ignorar la problemática que tiene el aprendizaje para los alumnos. Al respecto bay que resaltar la proliferación de estudios realizados en este ámbito en los últimos tiempos. Creemos interesante aprovechar dichos logros de cara no sólo a diagnosticar situaciones, sino para intervenir en ellas.

En este apartado quisiéramos resaltar la problemática didáctica que presenta el aprendizaje de esta parte de la Física en las aulas de ESO. Pero habría que señalar una serie de circunstancias, no específicas del módulo, pero aplicables al mismo; así, por ejemplo, podemos decir:

- El alumno no está acostumbrado a trabajar con material escrito que le plantee interrogantes que precisen un razonamiento; en otros niveles educativos las respuestas se buscan en el propio texto y no como fruto de una inferencia inmediatá.

- El alumno no suele establecer relaciones entre sus aprendizajes, no sólo en cuanto a los contenidos de diferentes lecciones sino muchas veces entre la «teoría», los «problemas" y, sobre todo, las experiencias de laboratorio.

- El alumno no valora de la misma forma el trabajo experimental que las explicaciones del profesor o el estudio del libro de texto, considerando más los aspectos lúdicos que los formativos de dicho trabajo.

- El alumno juega muchas veces a buscar la respuesta que quiere el profesor o a encubrit sus desconocimientos con contestaciones que no dicen nada; por ello, es preciso diversificar las fuentes de información para conocer cuáles son sus auténticas ideas y razonamientos. 
Figura 5

TEÓRIC O

\section{Te $\underline{\text { oxxí모 }}$ \\ - Mecàica newtoniana y galileana}

\section{Príine cipío}

- Es posible encontrar una ecuación o modelo matemático que represente 1 movimiento de un cuerpo

\section{Con $\underline{\text { wacepto }}$}

-espacio

- tiempo

-ecuación

-representación grafica -movimiento
¿Que relación ge puede establecer entre e $y$

tiempo empleat o?

¿Cual es la ecuación representativa de un movimiento rectilineo $y$ $\mathrm{unif}$ orme?

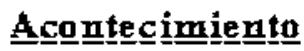

Estudio del mostimiento de una bola en nin tubo de aceite \{noviniento rectilineo y uniforme?

\section{PROCEDIMENTAL}

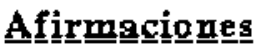

-Es posible crear una magnitud

(b) como relación entre e y

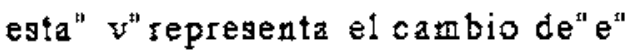
en el tiemo

- Las ecuaciones representativas del movimiento rectilineo $y$ uniforme son:

$$
\begin{aligned}
& e=v_{0}^{t}+e_{0} \\
& v=v_{0}=\text { cte. }
\end{aligned}
$$

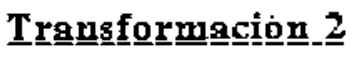

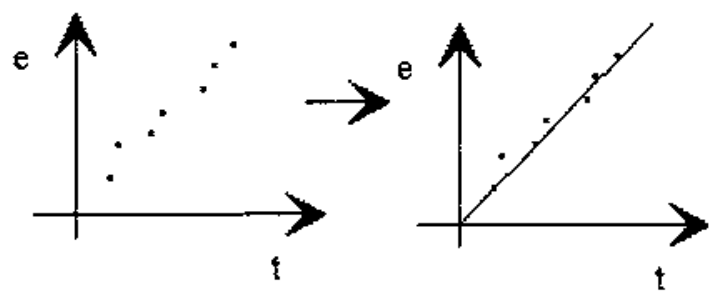

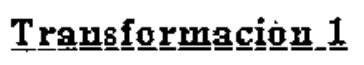

$$
t_{1}=\frac{\sum t_{t}}{3} ; t_{\mathcal{E}} \frac{\sum t_{\mathbf{y}}}{3} ; \cdots
$$

\begin{tabular}{|c|c|c|c|}
\hline espacio & \multicolumn{2}{|c|}{ tiempo } & Obseryadores \\
\hline$e_{1}$ & $t+1$ & ${ }^{\mathrm{t}} \mathbf{2}{ }^{\mathrm{i}} \mathbf{B}$ & \\
\hline$e_{2}$ & 2 & 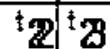 & \\
\hline
\end{tabular}

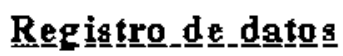


Figura 6

TEÓRICO

\section{Teㅡ으모므}

-Mecànica newtoniana

y' galileana

\section{Principio}

-Es posible encontrar una ecuación o modelo mate . tnatico que represente el movimiento de un cuerpo

\section{Cong $\underline{\text { ncepto }}$}

-espacio

- tiempo

-ecuación de $1^{\circ}$ y $2^{\circ}$ grado

-representación gràfica

- tangente a la curva

-velocidad

- movimiento

\section{PROCEDIMENTAL}

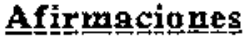

-Es posible crear una magnitud

(a) como relación entre v y t; esta" a"representa el canbio de" $v^{\prime \prime}$ en el tiempo

$¿^{C}$ ual es la ecuación representativa de un movithiento rectilitieo $y$ viliformenente acelerado?

\section{Transformación_ 3}

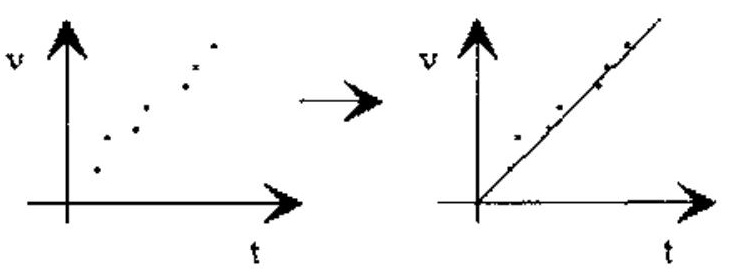

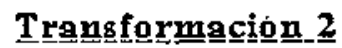

\begin{tabular}{|c|c|c|c|}
\hline$e_{1}$ & \begin{tabular}{|l|}
${ }^{t} 1$ \\
\end{tabular} & $\nabla_{1}$ & Ousenaines \\
\hline 42 & $t_{2}$ & $\nabla_{2}$ & \\
\hline
\end{tabular}

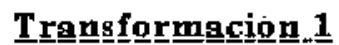
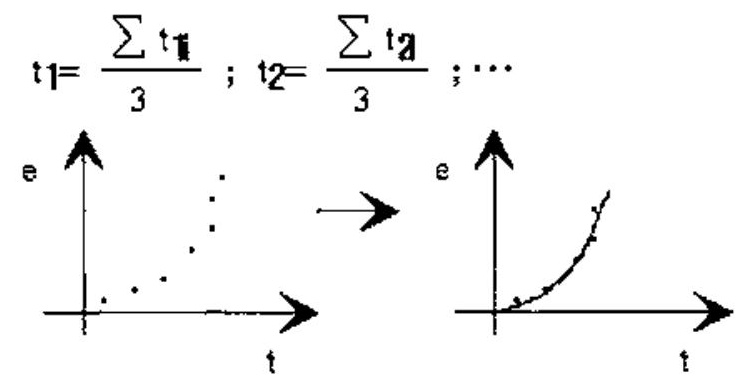

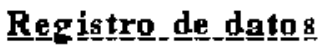

\begin{tabular}{|c|c|c|c|c|}
\hline espacio & \multicolumn{3}{|c|}{ tiempo } & observactones \\
\hline$\tau_{1}$ & $t \pi$ & ${ }^{t} \mathrm{Q}$ & ${ }^{t} B$ & \\
\hline${ }^{2} 2$ & ty & $t$ & tg & \\
\hline
\end{tabular}

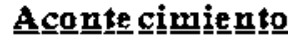

Estudic del movimiento de una bola en un plano inclinado(movimiento rectilíneo y uniformemente acelerado 
Con estas matizaciones, aplicables a otras partes de la materia, entramos en Ios contenidos específicos de este módulo de aprendizaje. Nos vamos a referir a los tópicos más relevantes. Algunos son fruto de trabajos exploratorios realizados con anterioridad (Pro 1984, Pro y Sánchez 1986 y García-Estañ et al. 1989), otros son contribuciones que aparecen en la literatura científica (Trowbridge y McDermott 1980,1981, Lovell 1982, Whitaker 1983, Aguirre y Erikson 1984, Hierrezuelo y Montero 1988, Laburú y Carvalho 1992, Sánchez et al. 1993) y lógicamente incorporamos los hallazgos de nuestra práctica diaria. Sin entrar en el origen de las ideas de los alumnos, ya estudiado por otros autores (Driver et al. 1989, Hierrezuelo y Montero 1989, Pozo 1992), las principales creencias que condicionan sus tazonamientos son los siguientes.

\section{Posición}

- Consideran equivalentes los conceptos de desplazamiento y espacio recorrido; muchas veces los confunden con el de trayectoria.

- Asocian el movimiento a aquellos objetos, cuerpos o sistemas que lo «pueden realizar» de forma mecánica o por sí mismos (un coche, una persona andando... pero no un árbol); incluso no creen que se mueva lo que «hay dentro" (un conductor, el reloj de pulsera del sujeto...).

- Les resulta difícil «salir» đe su sistema de referencia habitual al que consideran «reposo absoluto»e, incluso cuando lo Iogran, siguen pensando que «parece» que se mueve; tienen dificultades para asumir que un sistema está en reposo si se mueve con la misma velocidad que el sistema de referencia.

\section{Velocidad}

- La velocidad es siempre «espacio partido por tiempo».

- Identifican los $\mathrm{km} / \mathrm{h}$ pero tiene muchas más dificultades con los $\mathrm{m} / \mathrm{s}$.

- Confunder la velocidad con el movimiento; movimiento constante es aquél cuya velociđad es constante.

- No consideran el carácter vectorial de la velocidad; confunden velocidad con rapidez o celeridad.

- No ven clara la diferencia entre velocidad instantánea y velocidad media.

\section{Aceleración}

- Confunden aceleración con velocidad alta.

- La aceleración significa aumento de velociđad, por lo que frenar no es acelerar.

- Existe una gran đificultad para introducir el carácter vectorial de la aceleración.
- Tienen problemas con las unidades y, sobre todo, con el significado de segundo al cuadrado en el denominador.

\section{Gráficas y simbolismos matemáticos}

- Tienen dificultades para interpretar desde el punto de vista físico una gráfica, una ecuación o un resultado matemático.

- Confunden la trayectoria con la gráfica e/t.

- Interpretan las gráficas e/t y v/t de la misma forma.

- No ven la necesidad de que exista una proporcionalidad en las cantidades representadas en los ejes de coordenađas de las gráficas.

- Suelen tener problemas en la representación y, sobre todo, en las extrapolaciones de los datos no medidos; es muy habitual el «unir punto a punto», persistiendo este error a pesar de la insistencia reiterada del profesor.

\section{SELECCIÓN DE CONTENIDOS Y SECUENCIA DE ENSENANZA}

Todos los elementos anteriores nos permiten la selec. ción de unos contenidos de aprendizaje y la organización de los mismos en una secuencia de enseñanza. En la ta* bla I aparecen identificados los primeros. Sin embargo, dicha descripción no es suficiente para la clarificación de nuestra propuesta. Nos parece más adecuado el modelo de Needham (1987). Tomándolo como referencia se han elaborado los submódulos Reposo y movimiento (Fig. 7), Conceptualización de la velocidad (Fig. 8) e Iniciación al concepto de aceleración (Fig. 9).

En cada una de las figuras aparecen cuatro columnas. La primera representa esquemáticamente los submódulos ya reseñados y, junto a las fases del planteamiento constructivista, se pueden observar la secuenciación de los contenidos conceptuales.

La columna «Preguntas-clave» orienta sobre los interrogantes centrales de cada una de las actividades điseñadas. En ellos pueden detectarse algunos de los principios que inspiran nuestra propuesta:

- Importancia de la construcción de los aprendizajes a partir de las ideas de los alumnos y de la propia coheren. cia científica de los contenidos implicados (ver esque* mas conceptuales del análisis científico).

- Organización de contenidos de manera que permitan el establecimiento y secuenciación de relaciones conceptuales muy próximas (identificación, diferenciación y relación).

- Integración de los elementos teóricos y prácticos en el aula, dentro de un continuo que tiene el mismo marco de referencia. 
Tabla

\begin{tabular}{|c|c|c|}
\hline $\begin{array}{l}\text { CONTENIDOS } \\
\text { CONCEPTUALES }\end{array}$ & $\begin{array}{c}\text { CONTENIDOS } \\
\text { PROCEDIMENTALES }\end{array}$ & $\begin{array}{l}\text { CONTENIDOS } \\
\text { ACTITUDINALES }\end{array}$ \\
\hline $\begin{array}{l}\text { Cl.Sistemas de referencia. } \\
\text { Determinación de la po- } \\
\text { sición. }\end{array}$ & $\begin{array}{l}\text { Pl. Identificación de interrogantes } \\
\text { de la vida cotidiana. }\end{array}$ & $\begin{array}{l}\text { A.l.Interés por fenómenos o hechos } \\
\text { de la vida cotidiana. }\end{array}$ \\
\hline $\begin{array}{l}\text { C2.Espacio recorrido, trayec- } \\
\text { toria y desplazamiento. }\end{array}$ & $\begin{array}{l}\text { P2.Diseño y/o realización de expe- } \\
\text { riencias. }\end{array}$ & $\begin{array}{l}\text { A2. Rigor, precisión, orden,... en la } \\
\text { recogida de información. }\end{array}$ \\
\hline $\begin{array}{l}\text { C3. Velocidad media e instan- } \\
\text { tanea. }\end{array}$ & P3.Emisión de hipótesis & A3 Actitud critica y fundamentada. \\
\hline $\begin{array}{l}\text { C4. Estudio del movimiento } \\
\text { rectilineo y uniforme. }\end{array}$ & $\begin{array}{l}\text { P4.Observación y recogida de } \\
\text { datos cualitativos. }\end{array}$ & $\begin{array}{l}\text { A4. Asumir responsabilidades en } \\
\text { las tareas del grupo. }\end{array}$ \\
\hline $\begin{array}{l}\text { C5.Carácter vectorial de la } \\
\text { velocidad. }\end{array}$ & $\begin{array}{l}\text { P5.Utilización de aparalos de me- } \\
\text { dida. }\end{array}$ & $\begin{array}{l}\text { A5. Actitud flexible y de colabora- } \\
\text { ción en el grupo. }\end{array}$ \\
\hline $\begin{array}{l}\text { C6. Introducción al concepto } \\
\text { dc accleración. }\end{array}$ & $\begin{array}{l}\text { P6. Representación e interpretación } \\
\text { dc gráficas. }\end{array}$ & $\begin{array}{l}\text { A6. Valorar el conocimiento cienti- } \\
\text { fico como proceso de constric- } \\
\text { ción continua y de revisión } \\
\text { permanente. }\end{array}$ \\
\hline \multirow[t]{8}{*}{$\begin{array}{l}\text { C7.Estudio del movimiento } \\
\text { uniformemente acelerado. }\end{array}$} & $\begin{array}{l}\text { P7. Familarización con el cambio } \\
\text { de unidades y utilización del } \\
\text { S.I. }\end{array}$ & $\begin{array}{l}\text { A7. Fomentar una actitud positiva } \\
\text { hacia las Ciencias y sus reper- } \\
\text { cusiones sociales. }\end{array}$ \\
\hline & $\begin{array}{l}\text { P8. Comunicación e interpretación } \\
\text { de resultados. Elaboración de } \\
\text { conclusiones }\end{array}$ & A8.Mejorar la autoestima. \\
\hline & $\begin{array}{l}\text { P9. Expresión de ideas utilizando } \\
\text { un lenguaje científico adecua- } \\
\text { do. }\end{array}$ & A9.Divertirse en el trabajo. \\
\hline & $\begin{array}{l}\text { P10. Utilización de ecuaciones } \\
\text { matemáticas en la resolución } \\
\text { de probiemas. }\end{array}$ & \\
\hline & $\begin{array}{l}\text { P1 1. Intercambio y defensa de ideas } \\
\text { utitizando argumentaciones } \\
\text { fundamentadas. }\end{array}$ & \\
\hline & P12.Interpretación de textos. & \\
\hline & $\begin{array}{l}\text { P13, Determinacion de los errores } \\
\text { cometidos. }\end{array}$ & \\
\hline & P14.Revisión de lo aprendido. & \\
\hline
\end{tabular}

La tercera columna se refiere a los contenidos procedimentales que tratamos que el alumno aprenda a partir de las actividades desartolladas. En estos se pueden apreciar:

- Los que aparecen con mayor frecuencia son la expresión de ideas utilizando un lenguaje científico adecuado y la identificación de interrogantes de la vida cotidiana, contenidos fundamentales para alcanzar los objetivos generales del área en la educación secundaria.

- Aparecen procedimientos habituales (representación e interpretación de gráficas o utilización de ecuaciones matemáticas) pero al mismo nivel que otros menos usuales (intercambio y defensa de ideas utilizando argu- mentaciones bien fundamentadas, comunicación e interpretación de resultados, elaboración de conclusiones o revisión del aprendizaje).

- Implícitamente estamos considerando que bay actividades de aprendizaje diversas (trabajo de laboratorio, entre otros), pero no se ignoran otro típo de estrategias, tan formativas para los alumnos de estas edades.

- Hay presencia de contenidos como la emisión de hipótesis o diseño de experiencias por los alumnos, que tienen una mayor complejidad cognitiva por dos motivos: por un lado, por su propia naturaleza y, por otro, por la escasa presencia de este tipo de contenidos en la enseñanza institucional. 
Figura 7

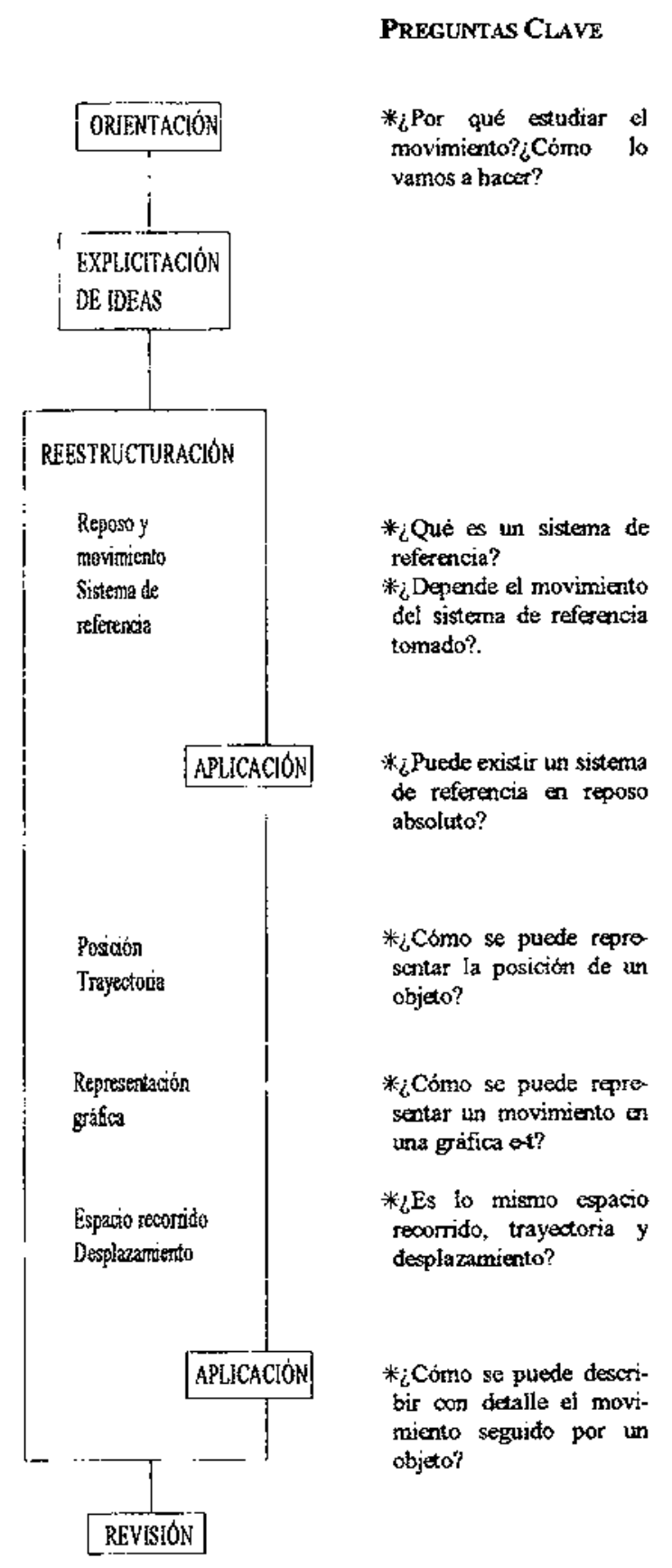

\section{CONTENmos \\ Procedimentaless}

* Identificación interrogantes vida cotidiana.

* Identificación de interro gantes vida ootidjama. *⿻丷木intepretactón de textos. * Intercambio y defensa de ideas.

*Expresión ideas utilizando lenguaje científico.

* Expresión ideas uritizando lenguaje científico. * Intercambio y defensa dc ideas.

* Representación e inte prdación de gráficas.

* Representación e interpretación de gráficas.

* Identificación de interrogantes vida cotidiana.

* Expresión de ideas utilizando lenguaje científico.

*Expresión de ideas utilfzando lenguaje científico. * Intercambio y defonsa de ideas utilizando argumentaciones bien fundamenladas

\section{INTENCIONES EDUCATIVAS}

* Iniciar y contextualizar la unidad didáctica. Motivar y organizar a los alumnos.

* Explicitar las ideas de los alummos sobre el movimiento

* Cuestionar su idea de moviniento.

* Crear la necesidad de establecer urs sistema de referencia.

* Aplicación conocimientos vida cotjdians.

* Deteminar la posición de un objedo con ayuda de unos ejes coorderados.

*Utilizar los graficos como elementos para represertar el movimiento.

*Relacionar y diferenciar los conceptos de desplazamiento y espacio reco. rido

* Aplicar los conceptos aprendidos a un caso concreto

* Evidenciar el cambio de las ideas inicisies en et alumno.
La cuarta columna, que hemos denominado «Intenciones educativas», perfila qué pretendemos en dicho momento diđáctico. También se vislumbran algunas đe las estrategias que proponemos:
- Partimos de la explicitación de ideas de los alumnos, con el fin de que éstos sean conscientes de sus propios conocimientos y sean capaces de diferenciarlos de los de otros compañeros (contraste entre iguales). 
Figura 8

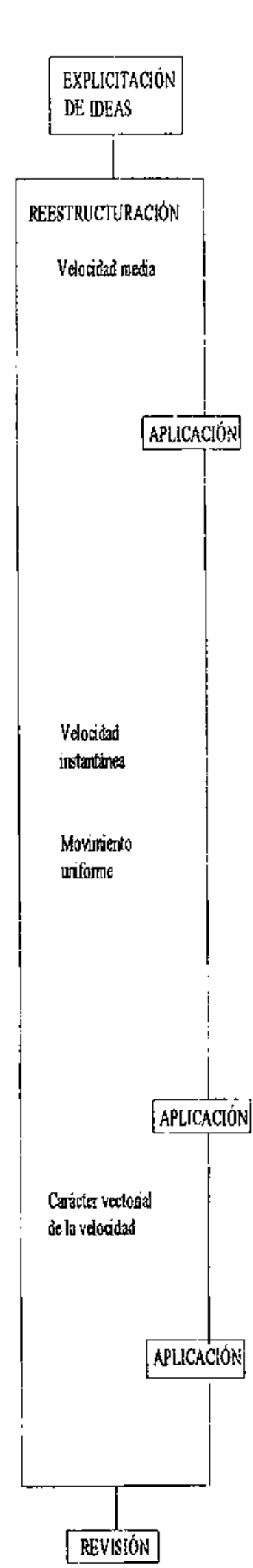

\section{Preguntas Clave}

* ¿Qué velocidad lleva un
objeto? récion exise
* ¿Qué relacion $_{\text {entre e, } v_{\mathrm{m}} \mathrm{y} \text { t? }}$

* Identiffcactón interro gantes vida cotidiana. *Expresión de jdeas utilizando lenguaje científico. * Intercambio y defensa ideas.

* Familiarización con el cambio de zaridades.

* ¿Cómo se puede determinar expecimentalmerte si un objeto se mueve oon velocidad constante? * ¿Qué problentatica conlleva al estudio real de un movimiento?

* ¿Es suficiente la $\mathrm{V}_{\mathrm{m}}$ para caracterizar un movimriento?.

* Q Qué información nos suministran las gáficas del M.R.U.?

* Cómo se puede estable cer la exuación represertativa de un M.R.U.?

* ¿Cómo se puede determinar la exalación representativa de un movimierto a partir de una gtafica?

* ¿Es suficiente un número para determinar la velocidad?

* LQué modificación implica la introducción de la direoción y el sentido de la velocidad a la resolución de problemas?

*Enisiór de hipótesis.

*Realización de experiarcias.

* Litilización de aparatos fe medida.

* Recogida de datos.

* Representación e irter. pretación de gráficas.

* Comunicación e interpretación de resultados.

* Valoración de exrores.

*Expresión de ideas utilizando lenguaje científico. * Intercambio y defeosa ideas.

*Representación e interpretación de gráficas.

* Comunicación e interpren tación de resultados.

*Ltitización de earacio nes matemáticas en la resolución de problemas. *Familiarización on ei cambio de unidades.

* Representación e interpretación de graficas * Resolución de proble mas.

*Utilizactón de exuaciones matemáticas en la resolución de problemas

* Identificación imlerrrogantes vidz coxidiana. * Expresión de jdeas utitizando lenguaje cientifico. * Utilización de ecuaciones matennáticas en la resolución de problemas

\section{INTENCIONES EDUCATIVAS}

* Explicitar las ideas de los alumnos respecto de la velocidad.

*Esctareser el concepto de velocidad media

* Medit el movimiento. Unidades y significado fisico. Realizar cálculos sercillos.

* Aplicar lo estudiado a un movinionto concreto (MRU). Iniciar al diseño y realización de experiencias. Aprender a caicular los enrores cometidos en las medidas * Moatrar la cokerencia interna del trabajo científico.

* Relacionar el nuevo concepto introducido $\left(V_{i}\right)$ con el anterior $\left(V_{m}\right)$.

* Relacionar los conceptos anteriores con las reprosentaciones gráficas.

* Cocestar la práctica con las equaciones represen-

tativas.

* Deducir las ecuaciones representativas del MR.U.

* Aplicar los conocimien. tos adquirides en la inter. pretación de graficas $y$ en ia resolución de pro. blenas.

* Coosiderar el caráder vectorial de la velocidad * Operar con vextores de forma elemental.

*Aplicación de lo estisdiado a una situación concreta

*Evidenciar el combio de ideas iniciales en el alumbo. 


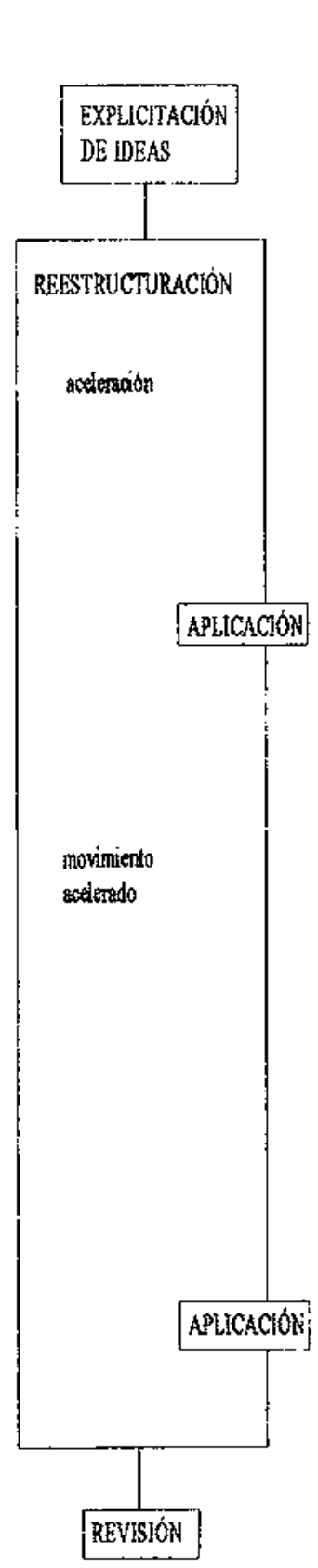

\section{Preguntas clave}

* ¿Qué debe ccurrir para que un objeto fleve acelo ración?

* ¿Como se puede calcular la aceleración

* ¿Cómo se puede determi. nar experimentalmente si un objeto se mueve oon velocidad variable?

* ¿Qué caracteriza las grificas de un M.RU.A.? * ¿Qué diferencia existe, desde el punto de vista de la representación gráfics, entre un M.RU y oro MR.U.A?

* Cómo se puede obtener la ecuación representstiva de un M.RU.A?

* ¿ Como se pueder tesolver problemss referentes al MRU.A.

\section{CONTENmos \\ Procedmentales}

\section{INTENCIONES} EDUCATTYAS

* Explicitar las jdeas de los alummos sobre la acelera. ciós.
*Idantificación de interrogartes de la vida cotidiana.

* Expresión de ideas utilizando ienguaje cientifioo. * Intercambio y defensa de ideas.

*Emisiór de hipótesis.

* Diseíto y realización de experiencias.

*Utitización de aparatos de medida.

* Recogida de datos.

* Comunicación e interpro tación de resultados.

* Representación e interpretación de gráficas.

* Interpreación de resultados.

* Intercambio y defensa ideas.

*Utilización de ecaracio nes maternáticas en la sesolución de probiemas. * Familiarización con el cambio de unidades. *Determinación errores.

*Utilizactón de ecuacio nes matemáticas en la resojución de problemas.
* Cuestionar sy ides de aveleración. Familiarizarse con su calculo y unidades
* Aplicar los conceptos adquiridos al estudio del movimiento acelerado. Familiariza al ahumo con el disefo y realización de experiencias * Mostrar la coherencia interna del trabajo cientifio.

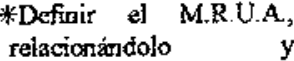
diferenciândolo del M.R.U.

* Conectar la práctica cors las ecuaciones representativas.

*Familiarizarse on las unidades y oon su cart? bio.

* Dextucir las ecuaciones representativas ded movimiento acelerado.

* Aplicar los conocinien. tos adquiridos en la interpretación de grificas y er la resolución de probletras

*Evidenciar el cambio de ias ideas iniciales an el alumeno.
- Tratamos de cuestionar aquellas ideas equivocadas a las que aludimos un el apartado anterior de este trabajo; otras veces simplemente se introduce nueva información pero tratando de justificarla o contextualizarla de cara a los alumnos.

- La construcción de los aprendizajes se hace relacionándolos, sin ambigüedades, con los ya existentes (importancia de crear estructuras conceptuales).
- Intentamos que se utilicen los conocimientos en nuevas situaciones, sin excluir los elementos matemáticos que sean necesarios, pero no reduciéndolos a éstos.

- Nos parece bastante formativo que el alumno perciba que "algo» se ha modificado tras el proceso de enseñanza (metaprendizaje).

Para la puesta en práctica de un planteamiento como el 
nuestro hay tres elementos importantes que no se pueden obviar. En efecto, la dinámica de la clase exige un trabajo sistemático en grupos donde sea posible el con. traste de ideas, el intercambio de opiniones, la realiza. ción de tareas conjunta y la reflexión crítica permanente sobre to que se va realizando. Esto exige un cambio en las funciones del profesor que, además de ser un facilitador de la información - no consideramos adecuado el descubrimiento autónomo-, debe guiar el proceso de aprendizaje, supervisar el trabajo diario de los alumnos, prestar atención a los progresos individuales dentro de los grupos y lógicamente estimularlos con problemas. Pero, sobre todo, tiene que creer en que esto puede funcionar.

Por otro lado, pensamos que los programas-guía pueden ser un buen formato para llevar al aula esta propuesta (Seminario de Física y Química, 1990). El planteamiento de un conjunto de actividades, mediante las cuales ponemos a los alumnos en la situación de elaborar conocimientos, de explorar alternativas, superando la mera asimilación đe conocimientos ya elaborados, facilita una estructura coherente, evitando un «aprendizaje» disperso, que dificulte que se establezcan relaciones conceptuales o relaciones entre el marco teórico y procedimental de los contenidos implicados.

Por último, creemos que el cuaderno de trabajo de los alumnos es un instrumento fundamental, tanto para el alumno como para el profesor. Para el primero porque le permite construir de forma personal su proceso de adquisición de conocimientos desde su propia experiencia y reflexión en el aula; lo que nos lleva a un aumento significativo de la participación, mejora de la expresión escrita, mejora de la capacidad de síntesis, promueve hábitos de trabajo de las ciencias... Para el profesor es un referente importante de cara al seguimiento y valoración de su secuencia de enseñanza, faceta clave para cumplir con las funciones de investigador de su acción educativa y de animador en el proceso de enseñanza y aprendizaje.

\section{REFERENCIAS BIBLIOGRÁFICAS}

AGUIRRE, J. y ERIKSON,G., 1984. Student's conceptions about the vector characteristics of three Physics concepts, J. Res. Sci. Teaching, 21, pp. 439-458.

DRIVER,R. et al. 1989. Ideas cientificas en la infancia y to adolescencia. (Madrid:Morata).

GARCÍA-ESTAÑ, R et al., 1988. El aprendizaje de la física en EGB. Exploración diagnóstica en Murcia. (ICE de la Universidad de Murcia).

HIERREZUELO, J. y MONTERO, A., 1989. La ciencia de los alumnos. Su utilización en la didáctica de la física y la química, pp. 39-66. (Laia: Barcelona).

HUERREZUELO, J. et al., 1991. Aprendizaje en física y quimica. (Elzevir: Vélez-Málaga).

LABURU, C. y CARVALHO, A., 1992. Investigación del desarrollo y aprendizaje de la noción de aceleración en adolescentes, Enseñanza de las Ciencias, 10, pp. 63-72.

LOVELL,K., 1982. Desarrollodeconceptos básicos matemáticos y científicos en los niños, pp. 95-136. (Morata: Madrid).

NEEDHAM, R., 1987. Teaching strategies for development understanding in Science. Children's learning in Science Project. (University of Leeds).

NOVAK, J. y GOWIN, D., 1988. Aprendiendo a aprender. (Martínez Roca: Barcelona).

POZO, I., 1992. Psicología de la comprensión y el aprendizaje de las ciencias. (MEC: Madrid).
PRO, A., 1984. Diseño, aplicación y evaluación de dos metodologias didacticas para la enseñanza de la física en un nivel elemental. Tesis doctoral. (Fac. Física: Sevilla).

PRO, A. y SÁNCHEZ, S., 1986. Aplicación de aprendizajes conceptuales a situaciones reales. Un estudio sobre los conceptos de Iongitud, tiempo y velocidad, Publicaciones, 9 , pp. 59-81.

SÁNCHEZ,G. y VALCÁRCEL, M.V., 1993. Diseño de unidades didácticas en el área de ciencias experimentales, Enseñanza de las Ciencias, 11, pp. 33-44.

SÁNCHEZ, J. et al. 1993. Detección de ideas previas en cinemática utilizado la composición de movimientos, Investigación en la Escuela, 19, pp.105-116.

SEMINARIO DE FÍSICA Y QUIMICA, 1990. La construcción de las ciencias físico-quimicas. Programas guía de trabajo. (Nau Llibres: Valencia).

TROWBRIDGE, D. y McDERMOTT, L., 1980. Investigation of student understanding of the concept of velocity in one dimension, American J. Physics, 48, pp.1020-1028.

TROWBRIDGE, D. y McDERMOTT, L., 1981. Investigation of student understanding of the concept of acceleration in one dimension, American J. Physics, 49, pp. 242.253.

WHITAKER, R., 1983. Aristotle is not dead: student understanding of trajectory motion, American J. Physics, 51, pp. 352-357. 


\begin{tabular}{|c|c|c|c|c|c|c|c|c|c|c|c|c|c|c|c|c|c|c|c|c|c|c|c|}
\hline Módulo: Cinemática & \multicolumn{6}{|c|}{ ¿En qué nivel deberia estudiarse? } & \multicolumn{5}{|c|}{$\begin{array}{l}\text { Grado importancia en estos } \\
\text { niveles }\end{array}$} & \multicolumn{5}{|c|}{ Dificultades de aprendizaje } & \multicolumn{7}{|c|}{$\begin{array}{c}\text { Recursos didácticos que usarias (máximo } \\
\text { (res) }\end{array}$} \\
\hline Unidades conceptuales $\downarrow$ & FEGB & PEGB & FEGB & POES & ress & $\begin{array}{ll}\operatorname{mos} 6 \\
\text { darie }\end{array}$ & 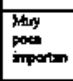 & \begin{tabular}{|l|}
$\begin{array}{l}\text { Poar } \\
\text { import }\end{array}$ \\
\end{tabular} & Nommal & 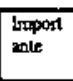 & $\begin{array}{l}\text { Muy } \\
\text { inpoer }\end{array}$ & 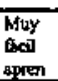 & 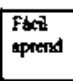 & Normal & \begin{tabular}{|l} 
Disel \\
aprend
\end{tabular} & 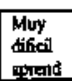 & 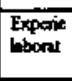 & $\begin{array}{c}\text { Explié } \\
\text { Prot }\end{array}$ & 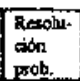 & 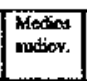 & 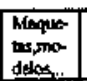 & 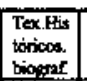 & $\begin{array}{l}\text { Vusing } \\
\text { sithes. }\end{array}$ \\
\hline Reposa y movimiento & & & & & & & & & & & & & & & & & & & & & & & \\
\hline Vestor de posición & & & & & & & & & & & & & & & & & & & & & & & \\
\hline Sistema de referencia & & & & & & & & & & & & & & & & & & & & & & & \\
\hline Trayectoria y desplazamiemo & & & & & & & & & & & & & & & & & & & & & & & \\
\hline $\begin{array}{l}\text { Velocidad media } \\
\text { Velocidad instantinean }\end{array}$ & & & & & & & & & & & & & & & & & & & & & & & \\
\hline Velocidad instanlinea & & & & . & & & & & & & & & & & & & & & & & & & \\
\hline Acelersción nestia & & & & & & & & & & & & & & & & & & & & & & & \\
\hline Aceleración instamánea & & & & & & & & & & & & & & & & & & & & & & & \\
\hline $\begin{array}{l}\text { Regresent. Graff.Mov. } \\
\text { Ec.Representativa del M.U. }\end{array}$ & & & & & & & & & & & & & & & & & & & & & & & \\
\hline $\begin{array}{l}\text { Ec.Representativa del M.U. } \\
\text { Ec.Repres.M.U.A }\end{array}$ & & & & & & & & & 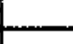 & & & & 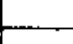 & 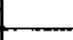 & 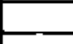 & & & & & & 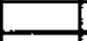 & & \\
\hline Essudiontivo obticuo & & & & & & & & & & & & & & & & & & & & & & & \\
\hline Essadio Mov. Onchuagetorio & & & & & & & & & & & & & & & & & & & & & & & \\
\hline Anglitud, Periodo, Frecuencia & & & & & & & & & & & & & & & & & & & & & & & \\
\hline Velocidad angol & & & & & & & & & & & & & & & & & & & & & & & \\
\hline Aceletrasi & & & & & & & & & & & & & & & & & & & & & & & \\
\hline Circular & & & & & & & & & & & & & & & & 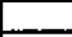 & & & & 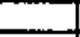 & 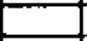 & & \\
\hline Acelelesacto & & & & & & & & & & & & & & & & & & & & & & & \\
\hline Acelerración nomal & & & & & & & & & & & & & & & & & & & & & & & \\
\hline
\end{tabular}

¿Qué criterios generales has utilizado para establecer el nivel al que deberian estudiarse los diferentes contenidos?

¿Cuáles son los errores conceptuales o las dificuttades más habituales que tienen tus alumnos respecto a esta unidad?

¿Utilizas alguna técnica o estrategia para detectarlos? 


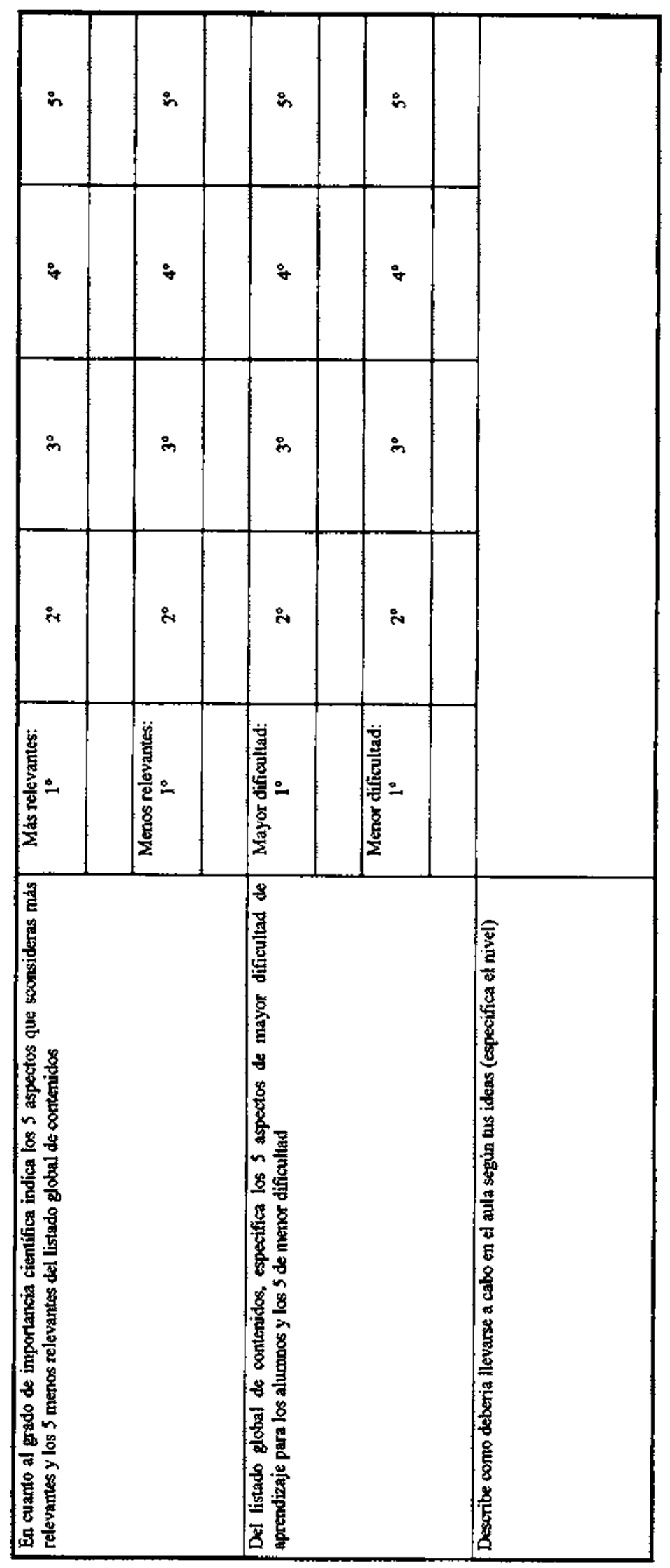

